\title{
In-vivo two-photon imaging of the honey bee antennal lobe
}

\author{
Albrecht Haase, ${ }^{1, *}$ Elisa Rigosi, ${ }^{2,3}$ Federica Trona, ${ }^{2}$ Gianfranco \\ Anfora, ${ }^{2}$ Giorgio Vallortigara, ${ }^{3}$ Renzo Antolini, ${ }^{1}$ and Claudio \\ Vinegoni $^{1,4}$ \\ ${ }^{1}$ Physics Department, University of Trento, Via Sommarive 14, \\ 38050 Povo (TN), Italy \\ ${ }^{2}$ IASMA Research and Innovation Center, Fondazione E. Mach, via E. Mach 1, \\ 38010 San Michele all'Adige, Italy \\ ${ }^{3}$ Center for Mind/Brain Sciences, University of Trento, Corso Bettini 31, \\ 38068 Rovereto, Italy \\ ${ }^{4}$ Center for Systems Biology, Massachusetts General Hospital, Harvard Medical School, 185 \\ Cambridge Street, \\ Boston, Massachusetts 02114, USA \\ albrecht.haase@unitn.it
}

\begin{abstract}
Due to the honey bee's importance as a simple neural model, there is a great need for new functional imaging modalities. Herein we report on the development and new finding of a combined two-photon microscope with a synchronized odor stimulus platform for in-vivo functional and morphological imaging of the honey bee's olfactory system focusing on its primary centers, the antennal lobes (ALs). Our imaging platform allows for simultaneously obtaining both morphological measurements of the AL's functional units, the glomeruli, and in-vivo calcium recording of their neural activities. By applying external odor stimuli to the bee's antennae, we were able to record the characteristic glomerular odor response maps. Compared to previous works where conventional fluorescenc microscopy was used, our approach has been demonstrated to offer all the advantages of multi-photon imaging, providing substantial enhancement in both spatial and temporal resolutions while minimizing photo-damages. In addition, compared to previous full-fiel microscopy calcium recordings, a four-fold improvement in the functional signal has been achieved. Finally, the multi-photon associated extended penetration depth allows for functional imaging of profound glomeruli.
\end{abstract}

(C) 2010 Optical Society of America

OCIS codes: $170.3880,170.2655,180.4315$

\section{References and links}

1. A. L. Hodgkin and A. F. Huxley, "Action potentials recorded from inside a nerve fibre" Nature 144, 710-711 (1939).

2. B. M. Salzberg, L. B. Cohen, and H. V. Davila, "Optical Recording of Impulses in Individual Neurones of an Invertebrate Central Nervous System,” Nature 246, 508-509 (1973).

3. G. Grynkiewicz, M. Poenie, and R. Y. Tsien, "A New Generation of Ca2+ Indicators with Greatly Improved Fluorescence Properties," J. Biol. Chem. 260, 3440-3450 (1985).

4. W. Denk, J. Strickler, and W. Webb, "Two-photon laser scanning fluorescenc microscopy," Science 248, 73-76 (1990).

5. K. Svoboda, W. Denk, D. Kleinfeld, and D. W. Tank, "In vivo dendritic calcium dynamics in neocortical pyramidal neurons," Nature 385, 161-165 (1997).

\#138064 - \$15.00 USD Received 12 Nov 2010; revised 3 Dec 2010; accepted 3 Dec 2010; published 16 Dec 2010

(C) 2011 OSA

1 January 2011 / Vol. 2, No. 1 / BIOMEDICAL OPTICS EXPRESS 131 
6. R. Menzel and M. Giurfa "Cognitive architecture of a mini-brain: the honeybee," Trends Cognit. Sci. 5, 62-71 (2001).

7. T. Franke, "In vivo 2-photon calcium imaging of olfactory interneurons in the honeybee antennal lobe," Dissertation, FB Biologie, Chemie, Pharmazie, Freie Universität Berlin (2009).

8. E. E. Lieke, "Optical recording of neuronal activity in the insect central nervous system: odorant coding by the antennal lobes of honeybees," Eur. J. Neurosci. 5, 4955 (1993).

9. C.G. Galizia, S. Sachse, A. Rappert, and R. Menzel, "The glomerular code for odor representationis species specifi in the honeybee Apis mellifera," Nat. Neurosci. 2, 473-478 (1999).

10. A. Gelperin and J. Flores, "Vital staining from dye-coated microprobes identifie new olfactory interneurons for optical and electrical recording," J. Neurosci. Meth. 72,97-108 (1997).

11. S. Sachse and C. G. Galizia, "The role of inhibition for temporal and spatial odor representation in olfactory output neurons: a calcium imaging study," J. Neurophysiol. 87, 1106-1117 (2002).

12. D. Flanagan and A. R. Mercer, "An atlas and 3-D reconstruction of the antennal lobes in the worker honey bee, Apis mellifera L. (Hymenoptera : Apidae)" Int. J. Insect Morphol. Embryol. 18, 145-159 (1989).

13. R. Brandt, T. Rohlfing J. Rybak, S. Krofczik, A. Maye, M. Westerhoff, H.-C. Hege, and R. Menzel, "Threedimensional average-shape atlas of the honeybee brain and its applications," J. Comp. Neurol. 492, 1-19 (2005).

14. B. Hourcade, E. Perisse, J.M. Devaud, and J. C. Sandoz, "Long-term memory shapes the primary olfactory center of an insect brain," Learn. Mem. 16 607-615 (2009).

15. C. G. Galizia and R. Menzel, "The role of glomeruli in the neural representation of odours: results from optical recording studies," J. Insect Physiol. 47, 115-130 (2001).

16. L. Moreaux and G. Laurent, "Estimating firin rates from calcium signals in locust projection neurons in vivo," Front. Neural Circuits 1, 2 (2007).

17. D. Müller, R. Abel, R. Brandt, M. Zöckler, and R. Menzel, "Differential parallel processing of olfactory information in the honeybee, Apis mellifera L.," J Comp Physiol A 188, 359370 (2002).

18. N. Yamagata, M. Schmuker, P. Szyszka, M. Mizunami, and R. Menzel, "Differential odor processing in two olfactory pathways in the honeybee," Front. Syst. Neurosci. 3, 16 (2009).

19. C. G. Galizia and R. Vetter, "Optical methods for analyzing odor-evoked activity in the insect brain," in Advances in Insect Sensory Neuroscience, T. A. Christensen, ed. (CRC press, 2004) pp. 349-392.

20. C. G. Galizia, S. L. McIlwrath, and R. Menzel, "A digital three-dimensional atlas of the honeybee antennal lobe glomeruli based on optical sections acquired using confocal microscopy," Cell Tissue Res, 295, 383-394 (1999) and http://neuro.uni-konstanz.de/23bee_morph/default.html.

21. P. Peele, M. Ditzen, R. Menzel, and C. G. Galizia, "Appetitive odor learning does not change olfactory coding in a subpopulation of honeybee antennal lobe neurons," J. Comp. Physiol. A Neuroethol. Sens. Neural Behav. Physiol. 192, 1083-1103 (2006).

22. G. Laurent, M. Wehr, K. MacLeod, M. Stopfer, B. Leitch, and H. Davidowitz, "Dynamic encoding of odors with oscillating neuronal assemblies in the locust brain," Biol. Bull. 191, 70-75 (1996).

23. S. Kirschner, C. J. Kleineidam, C. Zube, J. Rybak, B. Grunewald, W. Rössler, "Dual olfactory pathway in the honeybee, Apis mellifera," J. Comp. Neurol. 499, 933-52 (2006).

24. T. Faber and R. Menzel, "Visualizing mushroom body response to a conditioned odor in honeybees," Naturwissenschaften 88, 472-476 (2001).

\section{Introduction}

Important advances in neuroscience have always been strongly linked to the development of new investigative tools. While activity in single neurons has been detected for the firs time by electrical recording [1], later on, the development of a new class of voltage-sensitive dyes [2] has offered the possibility to optically image the functionality of neuronal circuits at both the single neuron and whole brain levels. In recent years the development of calcium-sensitive dyes [3] has provided another universal and sensitive method to study distinct information processing pathways in whole neural networks in particular when used in combination with two-photon laser scanning microscopy [4], allowing for in-vivo real-time monitoring of complex neural circuits down to several hundred micrometers within the specimen [5]. In this paper we focus our attention on two-photon calcium imaging of the honey bee (Apis mellifera) brain. With less than one million neurons, the honey bee is an excellent model for the study of neural systems of intermediate complexity [6], making it an ideal candidate for two-photon microscopy [7]. In the past years several optical imaging modalities have allowed to gain tremendous insights into the bee's olfactory system. After early pioneering works using voltage-sensitive dyes [8], various staining techniques have enabled to investigate different aspects of the odor process-

\#138064 - \$15.00 USD Received 12 Nov 2010; revised 3 Dec 2010; accepted 3 Dec 2010; published 16 Dec 2010

(C) 2011 OSA

1 January 2011 / Vol. 2, No. 1 / BIOMEDICAL OPTICS EXPRESS 132 
ing network by fluorescenc microscopy. In-vivo experiments using calcium-sensitive cellpermeant dyes have visualized the activity patterns of the glomeruli, the AL's functional units [9] and signals were found to be dominated by the Olfactory Receptor Neurons (ORNs) which are the input channels to the AL. Selective backfil staining with membrane-impermeable dyes [10] has instead allowed to record the AL's output signal from the Projection Neurons (PNs) [11]. In addition to the functional properties also the morphology of the olfactory centers has been studied extensively [12], more recently by confocal microscopy [13], and evidence of strong glomerular plasticity in the ALs has been reported lately [14]. While linear macro- and microscopy imaging techniques have been proven to be extremely successful in order to characterize this complex neuronal system, their intrinsic limitations have become more and more obvious [15]. Full-fiel microscopy does not offer sufficien axial resolution to resolve the exact origin of functional signals from deeper glomeruli and lacks the temporal resolution to determine whether valuable information might be encoded in the temporal structure of the recorded odor-evoked signals. Whereas confocal microscopy due to its intrinsic photo-damaging properties poses severe time constraints to in-vivo imaging sessions and has therefore only be used for morphological studies of the extracted brain.

Herein we report on the development and new finding of a neural imaging platform for functional imaging of the honey bee's antennal lobe's glomeruli. Our system permits to overcome the imaging impediments currently faced by neuroscientists. It enables us to acquire both in-vivo functional and morphological data of the ALs. Functional data show that we have been able for the firs time to longitudinally resolve active glomeruli, while the high temporal resolution permits a reconstruction of the neuron's firin rate [16]. Besides the well investigated T1 glomeruli which are projecting into the lateral antenno-cerebralis tract (1-ACT), the intrinsic two-photon optical penetration is deep enough to study the further classes of glomeruli that have not been optically accessible in the ALs yet, namely the T2, T3, and T4 glomeruli, projecting into the median antenno-cerebralis tract (m-ACT). Electrophysiological studies [17] and imaging of their axon terminals [18] suggest distinctive functional differences with respect to the l-ACT glomeruli.

\section{Methods}

Bees have been collected from outdoor hives and prepared in accordance to a well established protocol [19]. After chilling to immobility, bees were fi ed to a custom made imaging stage using dental wax (Kerr; Siladent). Then a small window was cut into the head's cuticula above the mushroom body, glands and trachea were gently moved aside, and a solution of calcium sensitive dye (fura2-dextran, Invitrogen) dissolved in 2\% Bovine Serum Albumin (Sigma-Aldrich) was injected by dye-coated micro-tips into the antenno-cerebralis tracts below the $\alpha$-lobe. Finally, the cuticula was carefully closed and the animals were stored for $20 \mathrm{~h}$ in a dark, cool, and humid place in order for the dye to diffuse into the AL. Before the imaging session, the cuticula, the glands, and the trachea above the $\mathrm{AL}$ were removed.

The experimental setup consists of a two-photon microscope (Ultima IV, Prairie Technologies) combined with an ultra-short pulsed laser (Mai Tai, Deep See HP, Spectra-Physics). The laser was tuned to $800 \mathrm{~nm}$ for fura-2 excitation. Dispersion was compensated by a pre-chirp module. Galvo-mirrors allowed for fast and variable scanning. All images were acquired with a water immersion objective (40x, NA 0.8, Olympus). Fluorescence is collected in reflection separated by a dichroic beam-splitter (Chroma Technology), filtere by a $70 \mathrm{~nm}$ band-pass filte centered at around 525nm, and detected by Photomultiplier tubes (Hamamatsu Photonics). A point spread function measurement verifie the microscope's resolution to be diffraction limited to $0.55 \mu \mathrm{m}$ in the plane and $2.6 \mu \mathrm{m}$ axially. Optimal signal-to-noise ratio was achieved with laser powers of about $10 \mathrm{~mW}$ on the sample without observing any induced photo-damage.

\#138064 - \$15.00 USD Received 12 Nov 2010; revised 3 Dec 2010; accepted 3 Dec 2010; published 16 Dec 2010

(C) 2011 OSA

1 January 2011 / Vol. 2, No. 1 / BIOMEDICAL OPTICS EXPRESS 133 
Temperature of the experimental environment was stabilized to $29^{\circ} \mathrm{C}$.
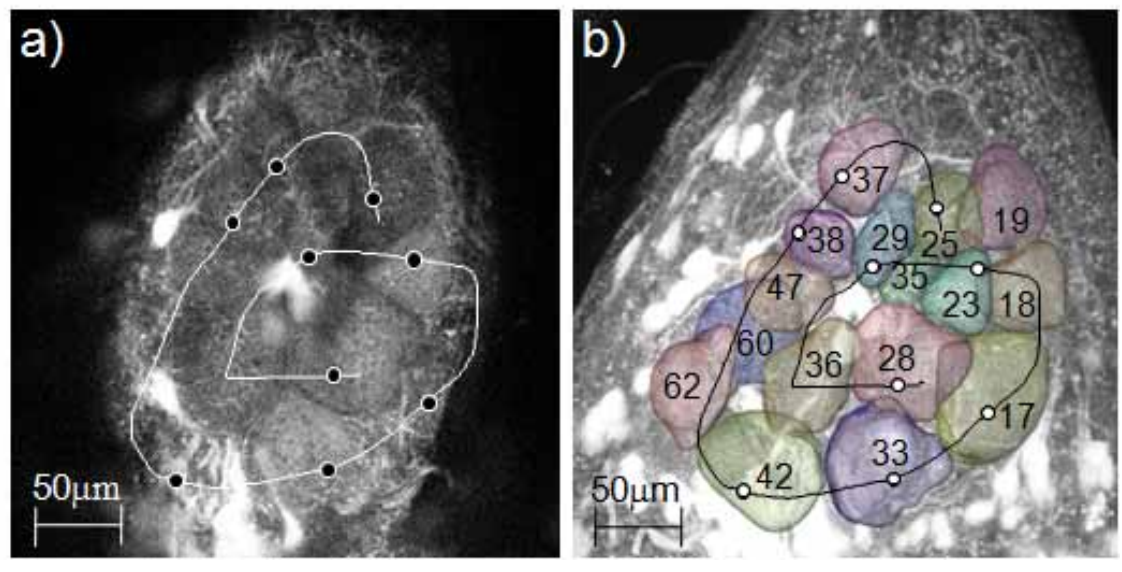

Fig. 1. a) Image of a right antennal lobe at $25 \mu \mathrm{m}$ depth: The line indicates the laser scanning trace, the dots label the measurement's reference positions corresponding to the vertical lines in Figure 2. (b) Axial projection view of the AL volume image stack, superimposed by the reconstructed surface plots of the involved $\mathrm{T} 1$ glomeruli, identifie and labeled according to [20].

A high functional temporal resolution of about $15 \mathrm{~ms}$ was obtained by laser scanning along one-dimensional custom-define traces, crossing the glomeruli of interest through an arbitrary horizontal plane [Fig. 1(a)]. All acquired data have been corrected for photo-bleaching, while $2 \mathrm{D}$ running-average filterin was used to reduce the noise level. Spatial averaging was performed over a typical glomerulus size of $30 \mu \mathrm{m}$, while temporal averaging was applied over $80 \mathrm{~ms}$ preserving all main dynamic features of the data.

A stimulus controller (CS-55, Syntech) delivered odor stimuli to the bee's antennae without changing the total air flu to avoid mechanical stimuli. The odor stimuli come from pasteur pipettes in which $10 \mu \mathrm{L}$ of an odor dissolved in mineral oil $(0.1 \mu \mathrm{g} / \mu \mathrm{L})$ are deposited on a piece of filte paper.

All command signals and acquisitions were controlled by a common gate which allows precise synchronization of the involved pulses. The experimental cycle began by starting the image acquisition. After $3 \mathrm{~s}$ the stimulus generater received a trigger releasing an odor puff of $2 \mathrm{~s}$ length. The exact arrival time of the odor at the bee antenna was measured and found to stable within $10 \mathrm{~ms}$, which allows e.g. accurate measurements of the neuronal response delay. After $9 \mathrm{~s}$ image acquisition stopped and automatic data evaluation started.

Thanks to the reduced photo-damage characteristics of the two-photon microscopy approach, due to the very limited absorption volume in the sample, the imaging sessions could be extended until 5 hours before we notice an essential drop in the brain activity.

Data analysis was automatically executed during the experiments by Matlab (Mathworks) scripts, while post-processing for 3D reconstruction, image segmentation, and volumetric measurements was performed using the software Amira (Visage Imaging).

\section{Results}

We firs recorded the spatio-temporal functional activity in the AL by measuring the two-photon calcium response signal along the line traces indicated in Figure 1 triggered by odor stimuli from three different flora components: 1-Hexanol, 1-Octanol, and 1-Nonanol. Enhanced neural 


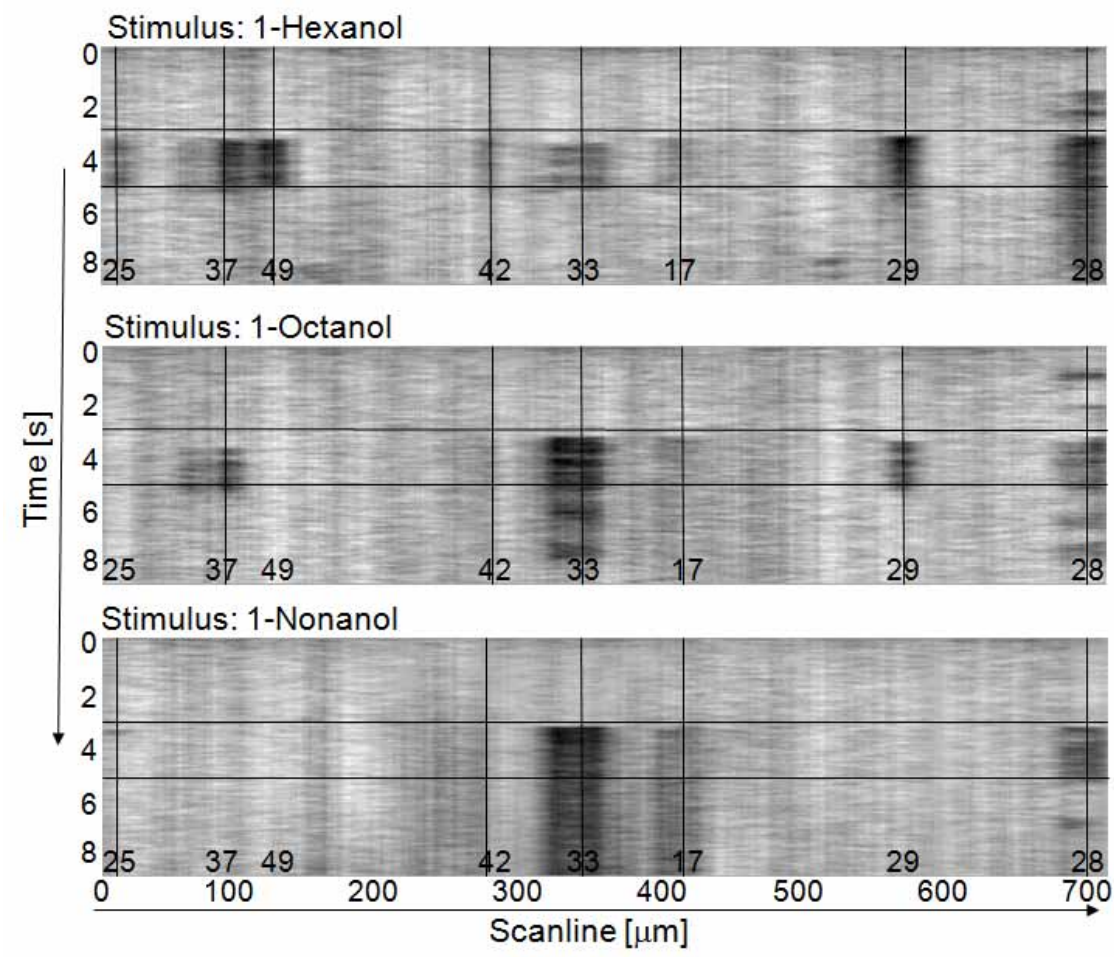

Fig. 2. Calcium response maps for three different odors (1-Hexanol above, 1-Octanol middle, 1-Nonanol below), recorded along the scanning trace in Figure 1. The stimulus period is enclosed by the horizontal lines, responding glomeruli centers are marked by vertical lines, numbers label the identifie glomeruli.

activity, leading to an increasing intra-neuronal calcium concentration, causes a drop in the measured two-photon fluorescenc intensity, producing dark bands in the scanlines-over-time maps at the positions of the corresponding glomeruli. We detected response signals of up to $20 \%$ intensity change, which is about 4 times higher than in comparable experiments using wide fiel imaging. The recorded maps are shown in Figure 2 and reproduce features which have already been observed by conventional single-photon fluorescenc microscopy [9], such as the very broad response of glomeruli T1-28, T1-33, and T1-17 to all tested odors. Likewise, 1Hexanol has been found to produce responses in several of the monitored glomeruli. Strikingly different from previously published data obtained with full-fiel microscopy [21] are the quite strong responses of glomeruli T1-29 and T1-37 for both 1-Hexanol and 1-Octanol. We have then exploited the larger penetration depth and the higher axial resolution offered by our setup, in order to obtain functional spatio-temporal odor response maps at different axial positions within the AL. Figure 3 shows the calcium response maps to an 1-Octanol odor stimulus at a depth of $25 \mu \mathrm{m}$ [Fig. 3(b)] and $50 \mu \mathrm{m}$ [Fig. 3(e)] respectively. The high axial resolution allows to clearly resolve the functional activity at the different depths. In particular the responses of the upper surface glomeruli T1-37 and T1-29, clearly visible at the imaging depth of $25 \mu \mathrm{m}$, disappear at $50 \mu \mathrm{m}$, while the weak response of the glomerulus T1-17 at $25 \mu \mathrm{m}$ becomes more pronounced at $50 \mu \mathrm{m}$. The temporal traces of the single glomeruli data [Fig. 3(c,f)] allow to analyze possible temporal components of the olfactory code, like response delay or oscillatory responses as reported in other animals [22]. 

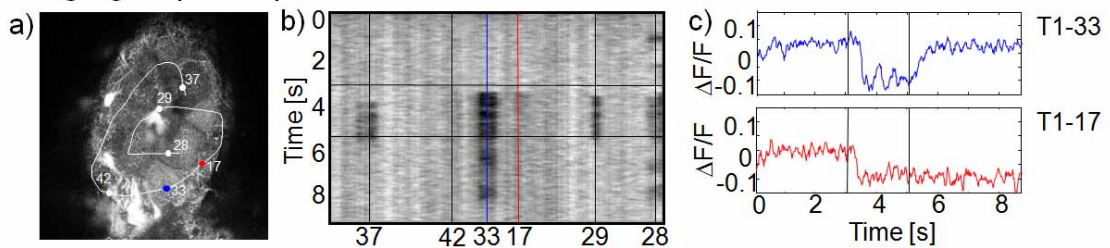

Imaging depth $50 \mu \mathrm{m}$
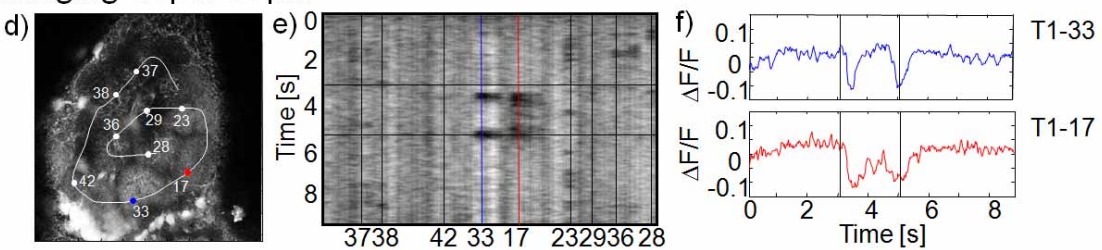

Fig. 3. Odor response maps to 1-Octanol at depths of $25 \mu \mathrm{m}$ and $50 \mu \mathrm{m}$ within the AL. (a) and (d) show the 2D images of the corresponding focal plane together with the line scan traces. In (b) and (e) the activity signal is plotted as a function of position along the line trace (x-axis) and as a function of time (y-axis). The stimulus period is enclosed by horizontal lines, the single responding glomeruli are marked by vertical lines. (c) and (f) show single temporal traces for the strongest responding glomeruli T1-33 and T1-17 at the two corresponding depths.

The maximum penetration depth for functional imaging was found to be $150 \mu \mathrm{m}$, while morphological data could be acquired from the complete ALs down to a depth of $400 \mu \mathrm{m}$. We restrict the presented data to a depth of $200 \mu \mathrm{m}$, to which we were so far able to identify glomeruli with certainty. In Figure 4 we present an image stack of $200 \mu \mathrm{m}$ representing the morphology of the glomeruli within the AL [Fig. 4(a)], together with the volumetric reconstruction of the glomeruli [Fig. 4(b)]. Green colored glomeruli belong to the T1 sensory tract projecting into the 1-ACT axonal tract, while blue and red colored glomeruli belong to the deeper laying T2 and $\mathrm{T} 3$ tracts, respectively, both projecting into the m-ACT. Of the latest ones most have never been optically accessible in-vivo because of their position below the surface or below the penetration depth limit of conventional microscopy.

The enhanced penetration depth and signal amplitude allowed also for the firs time optical recording of activity from those glomeruli of the T2 and T3 tract. In Figure 5 we show an example of these data. Figure 5(a) represents the glomerular response map to a 1-Hexanol stimulus at a depth of $150 \mu \mathrm{m}$. Apart from the standard post-processing methods this map has been averaged over 3 consecutive recordings with one minute recovery intervals in between. This allows to distinguish random fluctuation from specifi signal changes which becomes more crucial at that depth. In Figure 5(b) the corresponding focal plane together with the laser scan trace are visualized. The points where activity has been detected are marked by points. Comparing this points with our morphological reconstruction we could attribute all signals to specifi glomeruli which are labeled according to [20]. The green colored points represent signals from the $\mathrm{T} 1$ tract. In some of the cases the glomeruli are located in an upper layer above the imaging plane and the signal comes only from the few projection neurons connected to them. In these cases the absolute fluorescenc signal is very faint, but never the less a relative drop of up to $20 \%$ can be detected also here. The data points colored in blue and red mark glomeruli from the $\mathrm{T} 2$ and $\mathrm{T} 3$ tract, respectively. These glomeruli are located in the focal plane leading to a strong absolute fluorescenc signal. For the firs time we were able to optically record activity also 

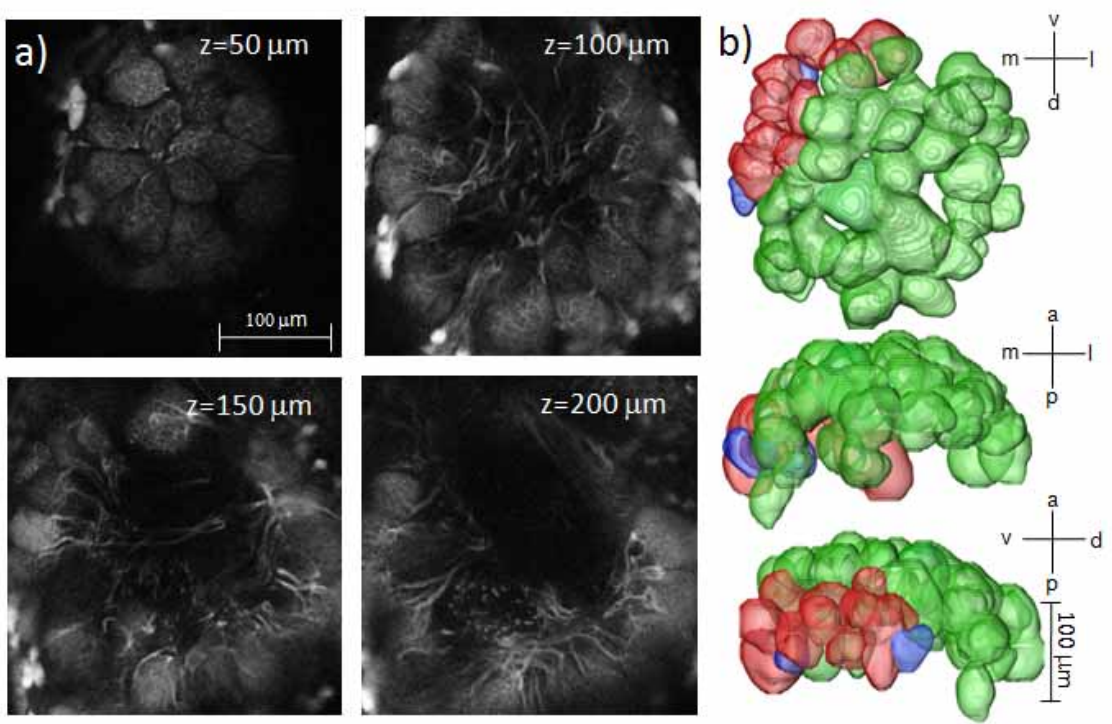

Fig. 4. a) Image stack examples down to $200 \mu \mathrm{m}$ penetration depth into the right AL. b) Reconstructed glomerular volume images in all projections, glomeruli colored in green are from the $\mathrm{T} 1$ tract projecting into the 1-ACT, the red glomeruli are from the $\mathrm{T} 3$ tract projecting into the $\mathrm{m}-\mathrm{ACT}$.

from these glomeruli deep within the antennal lobe. The T2 and T3 glomeruli project into the $\mathrm{m}$-ACT tract. This allows access to a whole new branch of the olfactory processing network, which so far could be investigated only partially by electro-physiological methods [17] or at the level of the axon terminals [18]. Figure 5(c) shows examples of single response curves for the T2 and T3 glomeruli. Strong activity signal and high temporal resolution are also here giving hope to a much needed systematical extension of the odor response maps to this glomerular classes.

\section{Conclusion}

The two-photon microscopy functional data we have presented in this work suggest that our imaging platform offers the capability to extend the specifi AL odor response maps that have been measured in the past for many different odor components and in most of the T1 glomeruli [9]. So far these maps contained only the static parameters response strength and consistency range, which might now be supplemented by adding temporal features as the response latency or oscillatory components. The intrinsic axial resolution and the extended imaging depth of two-photon microscopy has allowed us to resolve profound functional data. Odor response maps could therefore be completed by measuring the more profound classes of T2,T3, and T4 glomeruli.

This new possibility is of special interest because these glomerular classes are projecting into the m-ACT axonal tract and have been hypothesized to show fundamental differences regarding their odor coding properties $[17,18]$, as well as their memory related plasticity after odor conditioning [21]. The morphological division of the olfactory pathway into the two axonal tracts [23] seems to be accompanied by a complete functional division into two parallel processing branches. We have now for the firs time created optical access to the m-ACT branch in the antennal lobe which allows for a systematic study of their odor code. 
a) Stimulus: Hexanol, Imaging depth: $150 \mu \mathrm{m}$
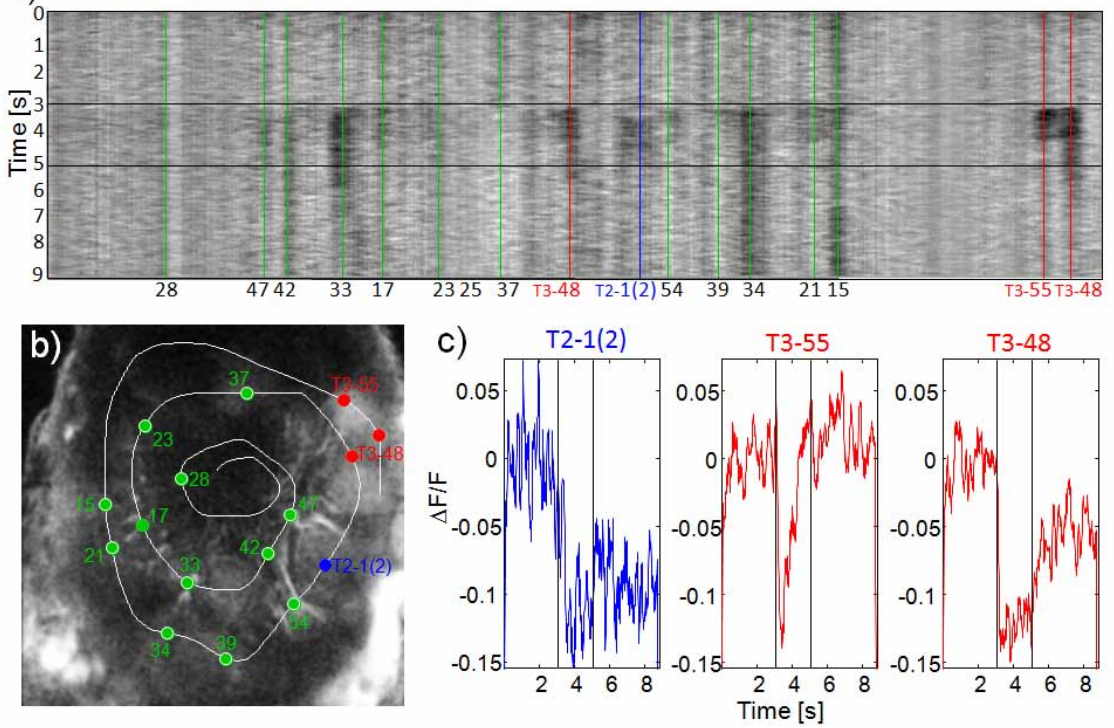

Fig. 5. a) Functional image map of responses to a 1-Hexanol stimulus recorded at $150 \mu \mathrm{m}$ depth from the left AL. b) The imaged focal plane together with the laser scan line. The responding centers are marked by points and labeled by the corresponding glomerular number. Green points correspond to T1, blue ones to T2, and red ones to T3 glomeruli. c) Single time traces of the responses from glomeruli of the T2 and T3 tract.

In addition we have obtained a 4-fold increase in the functional-related fluorescenc change with respect to similar experiments using wide fiel imaging. Another promising feature of a two-photon microscopy approach is the possibility to investigate sub-glomerular structures down to single neurons [7]. This becomes even more crucial if imaging is extended to higher order brain structures such as the mushroom body, where a meta-structure comparable to the AL's glomeruli is absent [24]. Finally, aside the resolution's improvements, the intrinsic twophoton limited photo-damage has offered extended imaging sessions up to 5 hours. This should allow in the future for in-vivo real time studies of the antennal lobe plasticity after odor conditioning [14].

\section{Acknowlegments}

We wish to thank T. Franke, J. Rybak, and R. Menzel for helpful discussions. C.V. acknowledges funding from the Provincia Autonoma di Trento (project COMNFI). 